\title{
Optically Beamformed Wideband Array Performance
}

\author{
Lluís Jofre, Senior Member, IEEE, Chrysavgi Stoltidou, Sebastián Blanch, Teresa Mengual, Borja Vidal, \\ Javier Martí, Member, IEEE, Iain McKenzie, and J. M. del Cura
}

\begin{abstract}
Optical beamforming networks (OBFNs) are an interesting alternative for the design of wideband antenna arrays, mainly due to their low losses and weight, their high parallel processing capabilities and their electromagnetic immunity. Nevertheless, for a practical implementation, different technological and architectural tradeoffs need to be assessed. In this paper, the performance of an OBFN system is analytically studied and experimentally demonstrated. The study forms part of the optical beamforming network project, a multigroup research project supported by the European Space Agency (ESA). Differently from bulky free-space geometries, the architecture is based on fiber-optic delay lines for the time delays generation and on a spatial light modulator for the phase shifts generation. Experimental results for an $\mathrm{X}$-band prototype are provided, showing beam steering accuracy, multibeam capability, amplitude distribution weighting and wideband operation.
\end{abstract}

Index Terms-Microwave photonics, optical beamforming, phased array antennas, spatial light modulator (SLM).

\section{INTRODUCTION}

$\mathbf{T}$ HE evolution of satellite communication and observation missions has shown a clear trend towards systems with higher performance resulting on higher complexity as well. More in particular, a key requirement for modern missions is the operation at wide bandwidths. As far as communication satellites are concerned, wide bandwidths are of great interest in order to accommodate broadband data connections, multiuser operation rates and wider communications coverage [1]-[3]. On the other hand, a wide bandwidth is also of great demand in observation missions in order to achieve high resolutions within a wide observation area [4]-[7]. As an example, modern synthetic aperture radar (SAR) systems-that represent a key instrument in satellite missions-such as COSMO-SkyMed [4], have been designed to provide resolutions in the range of $1 \mathrm{~m}$ and are able to scan up to $20^{\circ}$ in elevation. SAR systems of the

Manuscript received November 9, 2007; revised January 27, 2008. This work was supported in part by the European Space Agency through the OBEFONE project (OBEFONE: Technologies for Optical Payloads, ESA-SENER TRP EE-010) and in part by the Spanish CICYT projects TEC2004-04866-C04-02 and TEC2007-66698-C04-01.

L. Jofre and S. Blanch are with the Universitat Politecnica de Catalunya, Campus Nord, 08034 Barcelona, Spain (e-mail: luis.jofre@upc.es).

C. Stoltidou was with the Universitat Politecnica de Catalunya, Campus Nord, 08034 Barcelona, Spain. She is now with Consulting Ltd., Madrid, Spain.

T. Mengual, B. Vidal, and J. Martí are with the Nanophotonics Technology Center, Universidad Politecnica de Valencia, Camino de Vera, 46022 Valencia, Spain.

I. McKenzie is with the European Space Agency, European Space Research and Technology Centre, 2200 AG Noordwijk, The Netherlands.

J. M. del Cura is with the SENER, Ingeniería y Sistemas, Aerospace Division, Severo Ochoa, 4, 28760 Tres Cantos, Madrid, Spain.

Color versions of one or more of the figures in this paper are available online at http://ieeexplore.ieee.org.

Digital Object Identifier 10.1109/TAP.2008.923355 ultimate technology, such as the "phased array multifunctional radar" (PAMIR), are envisaged to demonstrate a very high resolution in the range of $\mathrm{cm}$ and wide scanning capabilities $\left( \pm 45^{\circ}\right)$ [5], [6]. Of course, all these require high-complexity hardware, wider bandwidths (in the range of $1 \mathrm{GHz}$ ), large number of transmit/receive (T/R) modules and the use of true time delay (TTD) units for scan control.

In this context, optical beamforming networks (OBFNs) are an interesting alternative to the design of wideband antenna arrays as they outmatch their microwave and digital counterparts offering attractive features, such as high parallel processing capabilities, low weight, immunity to electromagnetic interference and TTD function (i.e., beam squint - the beam steering angle dependence with frequency - may be avoided and wide bandwidths can be obtained).

Several optical beamforming architectures have been proposed until now using different technological implementations [8]-[19]. There are two main approaches to optically control phased-array antennas. TTD systems [8]-[12] provide large bandwidth but usually require long free-space sections that imply inevitably diffraction and loss issues, bounding this solution to the control of small antenna arrays. On the other hand, phase control systems [13] exploit the parallelism of free-space and so are normally more compact but pose critical beam squint problems, limiting this approach to small bandwidth applications.

Since most applications do not require full TTD control, a practical approach to control a N-element phase array consists on dividing the number $\mathrm{N}$ of elements of the array in a number, $N_{S}$, of $N_{e}$-element subarrays $\left(N=N_{S} \times N_{e}\right)$, and then assigning a phase shifter to each of the $\mathrm{N}$ antenna elements and a TTD to each of the $N_{S}$ subarrays. The combination of multistage free-space TTDs for the coarse pointing function and phase-shift control [14] for the fine pointing reduces the system cost and the beam squint problem for a given bandwidth. However, loss and high complexity issues — related with the number of multistage free-space sections-still limit the capability of the system to control large antenna arrays.

In order to overcome these limitations ESA initiated a study, through the so-called optical beamforming network (OBEFONE) project [15], aiming to the development and test verification of a high parallel, optical beamformer, scalable to the needs of a real application, such as a SAR antenna. During the OBEFONE project, different architectures were studied and the main advantages and disadvantages were extracted depending on the size-bandwidth constraints [15]-[19].

In this paper, an optimized approach-based on the combination of in-fiber implemented TTD units and phase-shift control units-to the design of optical beamformers is presented and demonstrated [18], [19]. Unlike former proposals based on 
free-space components for the implementation of TTD [14], the proposed beamformer provides TTD control at the subarray level using fiber optic delay lines (ODLs), and phase control with a short free-space section, exploiting the parallelism of collimated beams passing through spatial light modulators (SLMs). The architecture is able to control inter-element crosstalk and loss issues, keeping them under an acceptable level, and thus improving the scalability of the system for the control of large phased arrays, offering at the same time wideband squint-free operation.

In order to determine and study - both theoretically and experimentally - the constraints set by the interaction of the beamformer with an antenna array two basic analysis are performed. For the first one, a realistic 1280-subarray element as for the COSMO-SkyMed synthetic aperture radar antenna array is modelled. The design of the antenna model complies with the requirements set by modern radar applications (e.g., grating lobe level, sidelobe level, steering capability, etc.). Concisely, the array is divided into a number of subarrays both in the elevation and in the azimuth plane. Each radiating element is fed by a phase shifter from the T/R module and each subarray is steered by a TTD device. The number of subarrays or T/R modules depends on the considered plane.

For the second one, an $\mathrm{X}$-band $8 \times 8$ element antenna array (eight 8 -element subarrays) demonstrator has been designed and implemented. Experimental results for the radiation pattern measurements of the X-band demonstrator are provided, showing the capability for beam steering, amplitude distribution weighting, multibeam and wideband function.

Thus, the paper is divided into four main sections. Section II explains the operating principle of the beamformer and describes its architecture. In Section III, the main system parameters for the implementation of the OBFN in a real scenario are identified and assessed. Section IV presents the demonstrator experimental results for beam steering and tapered distributions capability, as well as multibeam and wideband TTD operation capability. Finally, the conclusions extracted are briefly summarized in Section V.

\section{OBFN ARCHITECTURE}

The proposed OBFN architecture, is based, as mentioned, on providing to the different subarrays time-delays introduced by fiber-optic delay lines (ODLs) and phase-shifts produced by a parallel-alignment spatial light modulator (PAL-SLM). Basically, the OBFN is modulating two RF signals $\left(f_{\mathrm{RF1}}\right.$ and $f_{\mathrm{RF} 2}$ ) into optical, which-after being amplified- are time delayed in the optical domain by the ODLs. Next, the signals are launched in free-space, by means of fiber collimators, where the PAL-SLM is used to introduce the appropriate phase shifts at every pixel-element. Finally, the optical signals are detected through photodiodes that converts them back to RF. We will first introduce the basic optical elements and then the whole beamformer structure will be described.

\section{A. Optical Time Delays and Phase Sift Subsystems}

Optical delay lines (ODL) may perform the physical delay using either electronically or mechanically controlled drivers.
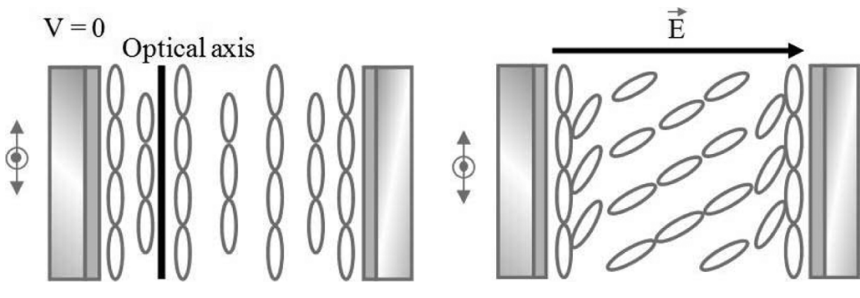

Fig. 1. PAL-SLM operating principle.

For the electronic version the programmable ODL has 8-bits of delay control from 0 to $560 \mathrm{ps}$ with a delay increment of $2 \mathrm{ps}$. In order to introduce time delays, the circuit structure consists of a series of crossbar switches that selectively route signals through a time delay element or through a bypass element.

On the other hand, the PAL-SLM is introducing the adequate phase shifts for the required beam steering, being controlled by specific voltage drive levels. The operating principle of the PAL-SLM relies on the control of the direction of its dielectrically anisotropic molecules alignment by means of the application of an external electrical field [20]. As a result, the polarization component of light that is perpendicular to the axis of its liquid-crystal molecules experiences a different refractive index depending on the applied voltage on each pixel. Instead, the polarization component of light that is parallel to the optical axis undergoes a constant refractive index. Therefore, if the optical carrier is aligned with this axis (see Fig. 1) the phase of the optical carrier can be continuously changed. However, if the sideband is polarized along the orthogonal polarization it will experience the constant refractive index and no phase shift will occur. In this way, the device is controlling the phase of the microwave signal by controlling the relative phase between optical carrier and sideband, as long as they have orthogonal polarizations.

Therefore, phase control using SLMs requires the use of a device that cross-polarizes the optical carrier and the sideband [18], [19]. Previous proposals [13], [14] mainly used acousto-optic modulators (AOM) to modulate a continuous wave-laser (CW-laser) and cross-polarize the optical carrier and its sideband. However, AOMs offer poor bandwidth, frequency range and modulation index compared to external modulators such as Mach-Zehnder modulators (MZM), widely available for telecom applications. Therefore, in the present beamformer architecture, a different approach was employed; birefringence can be used to cross-polarize two signals with a given wavelength spacing between them, using a differential group delay (DGD) module, which provides a group delay $t_{\text {DGD }}$ between two linear orthogonal polarization states.

The DGD is actually a high birefringent material. When light is launched to a birefringent material, it is broken up into a fast (the ordinary ray) and a slow (the extraordinary ray) component, standing for the two components of light experiencing the different refractive indices of the anisotropic material. As the two components travel at different velocities, the waves get out of phase after a certain distance. When the rays are recombined as they exit the birefringent material, the polarization state has changed as a result of this phase difference. The mentioned 


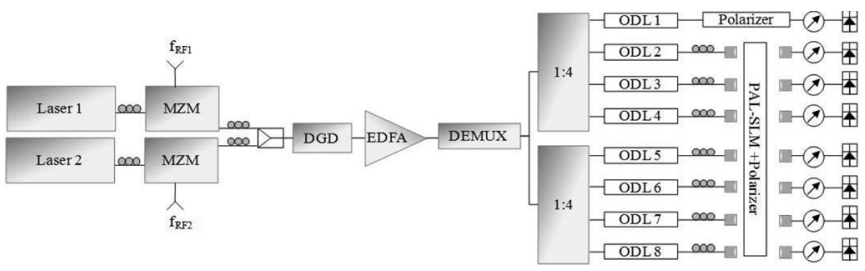

Fig. 2. Block diagram of a two-beam optical beamforming network for controlling an 8-subarray antenna.

phase difference at the output of the device depends on the wavelength. In other words, depending on the wavelength of the incident beam the birefringent crystal provides different polarization states at the DGD output.

If an amplitude modulated optical carrier is launched to a DGD and its polarization is linear at $45^{\circ}$ with the DGD axis, at the DGD output there is a polarization change with wavelength. The resultant phase shift between two wavelengths spaced at certain frequency $f_{\mathrm{RF}}$, caused by the DGD is given by

$$
\varphi=2 \pi f_{\mathrm{RF}} t_{\mathrm{DGD}}
$$

Consequently, in order to obtain linear orthogonal polarizations between the optical carrier $f_{o}$ and the sideband $\left(f_{o}+f_{\mathrm{RF}}\right)$, the birefringence needed is equal to $1 /\left(2 f_{\mathrm{RF}}\right)$. Thus, a DGD module that provides a $t_{\mathrm{DGD}}$ of $62 \mathrm{ps}$ has been used for the operating frequency of $8 \mathrm{GHz}$.

\section{B. Beamformer Transmission Operation}

sThe beamformer transmission architecture operation, Fig. 2, is as follows; first, two CW-lasers (to implement a beamformer of two independent beams) are amplitude modulated using a dual-drive MZM to generate SSB modulation [19]. Second, the signals are coupled, amplified by an Erbium Doped Fibre Amplifier (EDFA) and launched to a DGD module, which crosspolarizes the optical carriers and the sidebands. Next, the signals are demultiplexed using an add-drop multiplexer that routes the two different wavelength channels from the same data stream to two different paths [21], consisting on two $(1 \times 4)$ fibre-optic couplers/splitters. From each coupler, the signal is split into four channels wherein the time delays at subarray level are adjusted by means of optical delay lines (ODL). Next, the signals are launched to free-space by fiber collimators, where a PAL-SLM is used to control the phase-shift of every pixel element. After the PAL-SLM, a polarizer is needed to combine, in a single polarization state, the optical carrier and the sideband; otherwise no signal will be detected at the photodiodes. Additionally, as the phase-shift introduced by the PAL-SLM is relative, one of the channels does not pass through the PAL-SLM and the free-space polarizer; instead a fibre polarizer was placed in its path and the channel was used as a reference. Then, the beams are coupled from free-space into single-mode fibers, using once again fiber collimators. The amplitude of each channel is controlled by variable optical attenuators (VOA). Finally, the signals are photodetected by pigtailed photodiodes (optimized to operate at telecommunication wavelengths between 1310 and $1550 \mathrm{~nm}$ ). Assuming linear orthogonal polarizations for the optical carrier (e.g., $y$-axis) and the sideband (e.g., $x$-axis), the mi- crowave signal at the output of the photodiode is calculated to be

$$
I_{\mathrm{RF}}=-E_{s}^{x} E_{c}^{y} \sin (2 \beta) \cos \left(t w_{\mathrm{RF}}+\varphi_{\mathrm{PAL}-\mathrm{SLM}}\right)
$$

where $E_{s}^{x}$ and $E_{c}^{y}$ are the amplitude of the sideband and the optical carrier respectively, $\beta$ is the polarizer angle and $\varphi_{\mathrm{PAL}-\mathrm{SLM}}$ is the phase shift induced by the SLM on each pixel. Furthermore, polarization controllers are used to provide the adequate state of polarization to the MZMs, the DGD, and the PAL-SLM.

\section{OBFN SYSTEM PARAMETERS}

For the implementation of the OBFN, different technological and architectural aspects had to be assessed. Since antennas designated for next generation of X-band SAR systems need to provide an important number of different radiation pattern modes, a certain number of radiating elements is necessary. Taking the COSMO-SkyMed SAR antenna as an example, its beamforming network consists of 1280 transmit/receive (T/R) modules. For this range of elements, parameters such as the signal-to-noise ratio (SNR) and the crosstalk between the neighboring pixels are considered critical due to their impact in the performance of a large antenna array. For the respective studies the full 1280-subarray antenna array and the $8 \times 8$-element antenna demonstrator have been modelled.

\section{A. Signal Power Budget}

One of the critical parameters on a radar antenna is the sidelobe level ratio (SLR) that can be limited either by the array distribution weighting or eventually by the angularly distributed noise radiated by the different array elements. As the noise level in optical systems can reach critical levels, the assessment of the impact of the SNR in the OBFN radiation pattern performance is of vital importance. For this reason, as a first step, the effect on the array pattern of different SNR values for the two previous antenna models (the full array and the demonstrator) has been simulated to obtain the necessary minimum SNR threshold, $\mathrm{SNR}_{\mathrm{AF}}^{\mathrm{MIN}}$. Once the necessary SNR condition is known, it has been verified that both antenna models fulfils such conditions.

A simple approach to simulate the influence of the element noise in the Array Factor [22], [23] consists on modelling the total radiation pattern as the superposition of a "clean" array factor of $\mathrm{N}$ antenna elements plus a non-directional array pattern given by the noise level, resulting on a total signal plus noise factor $\mathrm{AF}_{(S+N)}$ given by

$$
\operatorname{AF}_{(S+N)}(\theta)=\sum_{n=0}^{N-1}\left(a_{n}+\delta(n)\right) e^{j\{n[k(d \sin \theta+\Delta l(n))+\alpha(n)]\}}
$$

being $\delta(n)$ a sample of a random variable following a Gaussian distribution of zero mean and a variance given by the SNR, $a_{n}$ the array coefficients, and $\Delta l(n)$ and $\alpha(n)$ the time delays and phase shifts respectively for the $n$th element, introduced by the OBFN.

Considering uncorrelated noise contributions of power $\sigma_{n}^{2}$ at each antenna element, the level of the signal with respect to the 


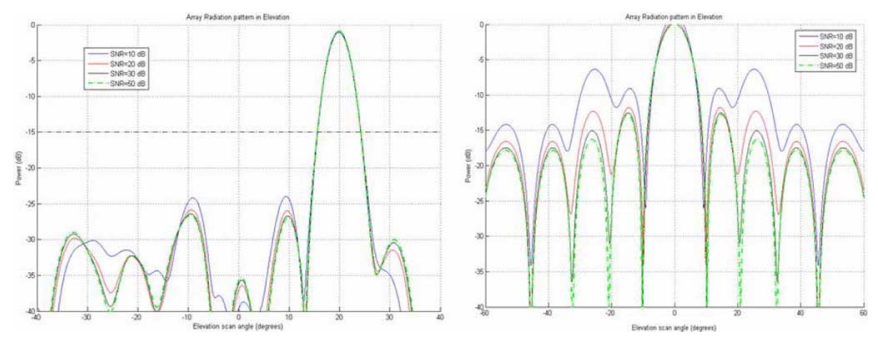

Fig. 3. Effect of the SNR on the array factor. Left: full array model (1280 elements). Right: demonstrator (8 elements).

noise increases by a factor N [24]. In other words, the SNR at the output - for a mean signal power $P_{s}$ and a mean noise power $P_{n}$ is given by

$$
\mathrm{SNR}_{\mathrm{AF}}=\frac{P_{s}}{P_{n}}=\frac{P_{s} N}{\sigma_{n}^{2}}
$$

Thus, the SNR for the array factor, $\mathrm{SNR}_{\mathrm{AF}}$, has, with respect to the SNR at the element level, $\mathrm{SNR}_{e l}$, an improvement given by the coherent sum of the antenna elements, $10 \log \left(N_{\text {pixels }}\right)$. Then the design condition can be expressed as

$$
\mathrm{SNR}_{\mathrm{AF}}=\mathrm{SNR}_{e l}+10 \log \left(N_{\text {pixels }}\right)>\mathrm{SNR}_{\mathrm{AF}}^{\mathrm{MIN}}
$$

where $\mathrm{SNR}_{e l}$ is the SNR of each antenna element at the output of the OBFN, just after photodetection.

According to (5), for the 1280 elements array there would be a gain $(10 \log N)$ of $31 \mathrm{~dB}$ in the SNR of the array radiation pattern, with respect to the SNR of each antenna element. For the 8 -subarray demonstrator this gain is only $9 \mathrm{~dB}$ and the influence of the $\mathrm{SNR}_{e l}$ in the array is consequently bigger. In Fig. 3 the simulation results for the impact of the different SNR values in the array pattern for the model antennas are illustrated. As seen from the graphs, it is clear that in order to have a pattern clean enough to identify the $-40 \mathrm{~dB}$ sidelobes, an $\mathrm{SNR}_{\mathrm{AF}}^{\mathrm{MIN}}$ around $50 \mathrm{~dB}$ would be necessary. Using $\mathrm{SNR}_{\mathrm{AF}}^{\mathrm{MIN}} \geq 50 \mathrm{~dB}$ in (5) results on a $\mathrm{SNR}_{e l}$ higher than $20 \mathrm{~dB}$ for the realistic SAR antenna model ( $N=1280$ elements $)$ and an of at least $40 \mathrm{~dB}$ for the demonstrator model ( $N=8$ elements).

To verify the accomplishment of these criteria we introduce the physical parameters of the OBFN system into the expression of the SNR of one antenna element at the output of the photodetector

$$
\mathrm{SNR}_{e l}=\frac{I_{e l}^{2} Z_{0}}{I_{n}^{2} Z_{0}+4 k T_{0}(F-1) B}
$$

where $Z_{0}$ represents the input impedance of the antenna element, the first and the second term of the denominator represent the shot noise and the thermal noise respectively, and $I_{e l}$ and $I_{n}$ are the detected current at each photodiode and the shot-noise
TABLE I

OPtical Power AND ATTENUATION AT THE OBFN DEMONSTRATOR

\begin{tabular}{|l|l|l|}
\hline Factor & Correspondence & Value \\
\hline$P_{\text {source }}$ & optical source power & $10 \mathrm{dBm}$ \\
\hline$I L_{M Z M}$ & losses in the Mach-Zehnder Modulators & $9 \mathrm{~dB}$ \\
\hline$I L_{M Z M}$ & losses in the polarizer & $2 \mathrm{~dB}$ \\
\hline$I L_{M Z M}$ & losses in the DGD device & $2 \mathrm{~dB}$ \\
\hline$I L_{M Z M}$ & losses in the PAL-SLM & $6 \mathrm{~dB}$ \\
\hline$I L_{M Z M}$ & losses in the time delay sections & $1.5 \mathrm{~dB}$ \\
\hline$I L_{M Z M}$ & losses in the demultiplexer & $1 \mathrm{~dB}$ \\
\hline$I L_{M Z M}$ & losses in the optical attenuators & $1 \mathrm{~dB}$ \\
\hline $10 \log N$ & demultiplexion power reduction & $9 \mathrm{~dB}$ \\
\hline
\end{tabular}

current given respectively by (7) and (8), shown at the bottom of the page.

In these equations, $R_{\text {in }}$ is the intrinsic current responsivity, $n$ is the quantum efficiency, $M$ is the multiplication ratio, $F$ is the excess noise factor and $P_{c}$ and $P_{s}$ are the power of the carrier and the sideband signal respectively. Both signals are suffering the attenuation of the different optical stages. Consequently, the power of the beams, just before photodetection, will be

$$
\begin{aligned}
P_{c}=P_{s}= & P_{\mathrm{Source}}-I L_{\mathrm{MZM}}-I L_{\mathrm{pol}}-I L_{\mathrm{DGD}}-I L_{\mathrm{SLM}} \\
& -I L_{\mathrm{ODL}}-I L_{\mathrm{DEMUX}}-I L_{\mathrm{VOA}}-10 \log N .
\end{aligned}
$$

The level of the optical power or loss at each stage is analytically explained in Table I. As the insertion losses of the system are high, a $19 \mathrm{~dB}$ gain EDFA was included in the design of the optical part to compensate for these losses. For those values and by using low noise avalanche photodiodes (APDs), SNR at each antenna element results in the order of $40 \mathrm{~dB}$.

In order to verify the experimental SNR level at the output of the system the mean power level of a signal at the output of a photodetector has been measured to be $-29 \mathrm{dBm}$, which - considering that the noise spectral density and the noise floor of the system are $-161 \mathrm{dBm} / \mathrm{Hz}$ and $-71 \mathrm{dBm}$ respectively-implies an SNR of $42 \mathrm{~dB}$ for a bandwidth of $1 \mathrm{GHz}$.

\section{B. Beam-Channel Crosstalk}

The compactness of the PAL-SLM in introducing the individual phase shifts for the different elements of the array is based on the high level of parallelism obtained by the free-space operation principle consisting on a set of parallel collimated beams generated at the ODL outputs passing through the pixeled PAL-SLM and finally detected at the photodetector inputs. Since optical beams diverge as they propagate, interference between adjacent beams may appear either in the ODL-SLM paths or in the SLM-photodetector paths. As a result, part of the power

$$
\begin{aligned}
I_{e l} & =R_{\text {in }} n M \sqrt{2 P_{c} P_{s}} \\
I_{n} & =\sqrt{2 F q R_{i} \eta M^{2}\left(P_{c}+P_{s}+P_{b}\right) B+2 q\left(I_{d s}+F M^{2} I_{d b}\right) B} .
\end{aligned}
$$




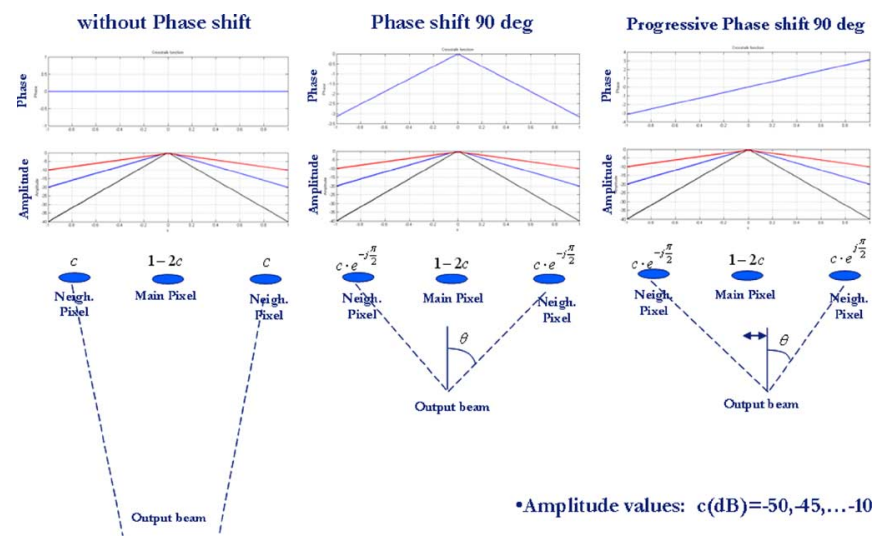

Fig. 4. Crosstalk Models. Left: $0^{\circ}$ phase shift. Center: $90^{\circ}$ symmetrical phase shift. Right: $90^{\circ}$ progressive phase shift.

that is propagated by one beam can illuminate the photodetectors of its adjacent pixels, leading to a phenomenon known as beam crosstalk. Crosstalk is a critical parameter of the system due to the fact that interference may change original amplitudes or phases and affect the performance of the antenna array in terms of beam pointing errors, main lobe level reduction and grating lobe level variations. Assuming that the aperture size of the photodetector is smaller compared to the beam footprint, a simplified crosstalk model has been studied during the system design analysis, which considers the contribution to one particular pixel, n, with complex coefficient (amplitude and phase) $I_{e l}(n)$, from the two closest beam neighbors with complex coefficients $I_{e l}(n-1)$ and $I_{e l}(n+1)$ given by the crosstalk geometry. Depending on the geometry of the crosstalk (longitudinal and lateral position of the beam source with respect to the pixel affected by the crosstalk), the contribution from the neighboring beams could have a symmetrical or asymmetrical phase shift effect. Fig. 4 shows the three cases that have been considered; the first case (Fig. 4, leftmost) considers no phase shift between the neighboring pixels, a second case (Fig. 4, center) assumes a symmetrical phase shift of $90^{\circ}$, while a third case (Fig. 4, rightmost) introduces a progressive phase difference of ${ }^{9} 0$ o.

Being $A F(\theta)=\sum_{n=0}^{N-1} I_{e l}(n) e^{j\{n[k(d \sin \theta+\Delta l(n))+\alpha(n)]\}}$ the array factor without crosstalk, the array factor affected by crosstalk can be expressed as

$$
A F_{x t a l k}(\theta)=\sum_{n=0}^{N-1} I_{e l}^{\prime}(n) e^{j\{n[k(d \sin \theta+\Delta l(n))+\alpha(n)]\}}
$$

where the signal at the n-element is modelled as

$$
I_{e l}^{\prime}(n)=\left(1-2 c_{e l}\right) I_{e l}(n)+c_{e l} I_{e l}(n-1)+c_{e l} I_{e l}(n+1)
$$

being $c_{e l}$ the crosstalk coefficient. In the simulations it has been modelled the effect of a crosstalk level $\left(\cong 20 \log c_{e l}\right)$ of the order of $-5,-10,-20$ and $-40 \mathrm{~dB}$, for the case of the 1280element model antenna. Figs. 5-7 show the resulting radiation patterns.
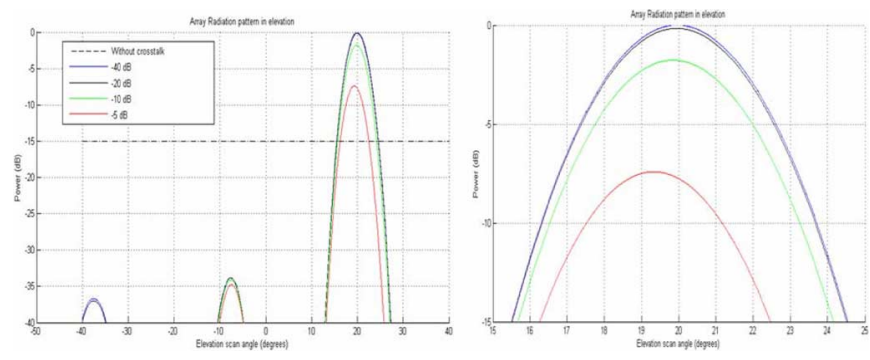

Fig. 5. Array in elevation with uniform phase crosstalk. Left: grating lobes and main lobe. Right: main lobe detail.
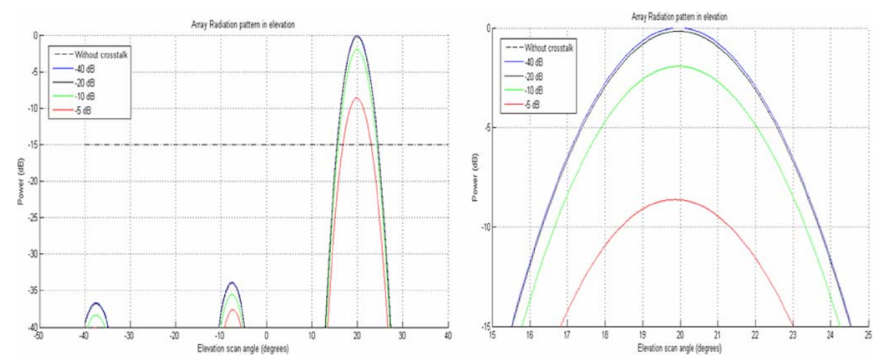

Fig. 6. Array in elevation with a symmetrical phase shift of $90^{\circ}$ crosstalk. Left: grating lobes and main lobe. Right: main lobe detail.
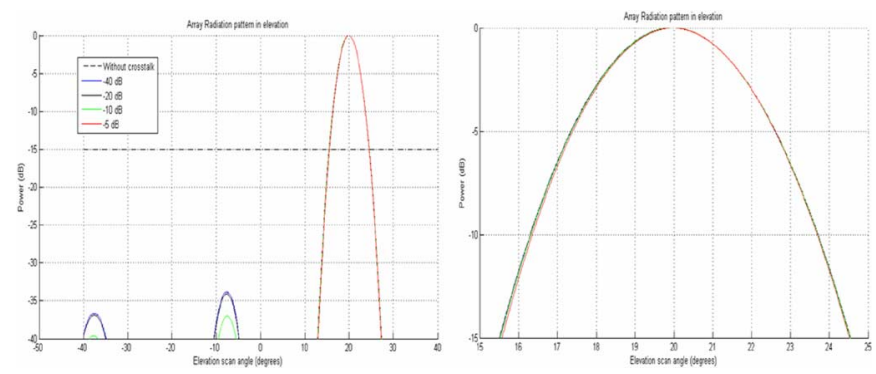

Fig. 7. Array in elevation with a progressive phase shift crosstalk. Left: grating lobes and main lobe. Right: main lobe detail.

As can be seen from Figs. 5-7, the effect of crosstalk in the array performance depends on the phase shift between the adjacent pixels. In particular, for the first two cases (with uniform phase and with a symmetrical phase shift of $90^{\circ}$ ) similar main beam losses occur. On the other hand, with a progressive phase of $90^{\circ}$ smaller main beam losses but more significant beam disappointments - not too visible on the figure-will appear.

Consequently, crosstalk has a direct impact especially in the main lobe level, particularly with crosstalk levels of above $-20 \mathrm{~dB}$. Interference at the order of $-40 \mathrm{dBs}$ leads to a practically null effect, while a crosstalk level of $-20 \mathrm{~dB}$ decreases the power of the main lobe by less than $0.5 \mathrm{~dB}$. Thus, a crosstalk level of $-20 \mathrm{~dB}$, which implies proportionally $-20 \mathrm{~dB}$ in the optical domain as well [17], could be considered the minimum acceptable for all the different crosstalk models, in order to comply with the specifications of the SAR antenna array. Nevertheless, the desirable crosstalk for a practical null interference sets the limits under the level of $-40 \mathrm{~dB}$.

The crosstalk level was taken into account in order to determine in the OBFN the optimum PAL-SLM configuration. In particular, it has been calculated the minimum pixel-to-pixel distance to have a beam-channel crosstalk below $-20 \mathrm{~dB}$. Con- 


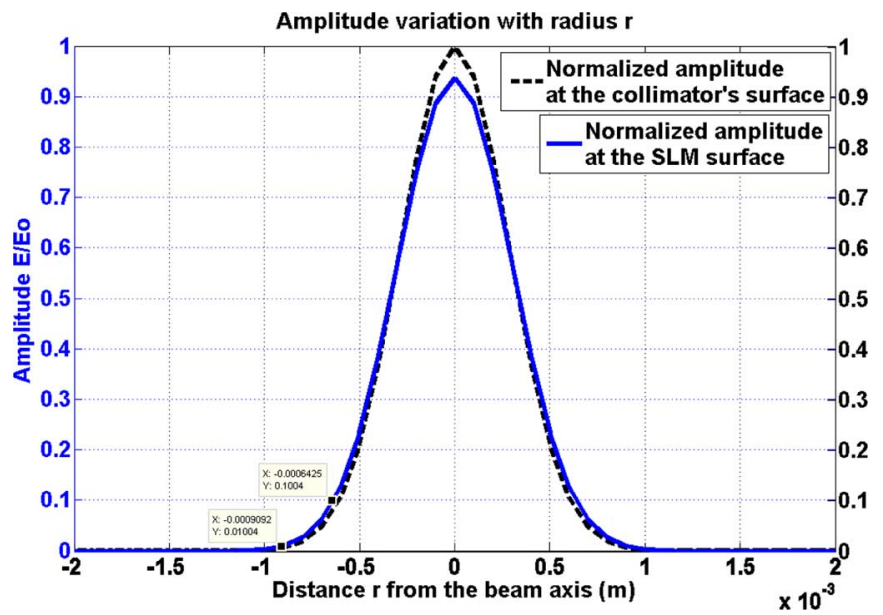

Fig. 8. Normalized electric field amplitude variation, with the radius $r$ : (dashed) at the collimator surface for $z=0 \mathrm{~mm}$, (solid) at the SLM surface for $z=120 \mathrm{~mm}$.

sidering Gaussian beams, the electric field at a radial distance $r$ from the beam centre and a distance $z$ from its waist, is given by

$$
E(r, z)=E_{o} \frac{w_{o}}{w(z)} e^{-r^{2} / w^{2}(z)} e^{-i k z-i k r^{2} / 2 R(z)+i \zeta(z)}
$$

where $E_{o}$ is the electric field amplitude, $w_{o}$ is the beam waist, $w(z)$ is the beam width, $R(z)$ is the radius of curvature and $\zeta(z)$ is the longitudinal phase delay. The electric field variation with radius $r$ was represented at a distance $z=0 \mathrm{~mm}$ (Fig. 8, dashed) and a distance $z=120 \mathrm{~mm}$ from the source beam (Fig. 8, solid). As seen from the figure, at $z=120 \mathrm{~mm}$ along the beam, the fall of the electric field by $20 \mathrm{~dB}$ or $40 \mathrm{~dB}$ (that is, by $10 \%$ and $1 \%$ of its maximum value) takes place at $r_{c_{20}}=0.6 \mathrm{~mm}$ or $r_{c_{40}}=0.9 \mathrm{~mm}$ respectively away from the collimated beam centre. Thus, configuring adequately a PAL-SLM of many pixels, to implement a mask of "super-pixels" having a diameter of $D \geq 2 r_{c}$ the crosstalk can be kept under the level of $-20 \mathrm{~dB}$. In the case of the OBFN, the PAL-SLM used (CRI-SLM-128-P-NM) incorporates 128 pixels of pitch size of the order of $100 \mu \mathrm{m}$. This means that for the control of an antenna array of 8 elements (with one path channel kept as a reference), which is the case of the experimental demonstrator, the pixels of the SLM can be grouped to implement a "super-pixel" having a pitch size of up to $1.8 \mathrm{~mm}$. In this way, the beam divergence from the SLM to the output collimator is reduced significantly while the crosstalk of the system from the input collimator to the SLM is practically null $(-40 \mathrm{~dB})$ as the spot of each collimated beam that impinges in the SLM mask is of the order of the "super-pixel" pitch-size (Fig. 9).

\section{EXPERIMENTAL RESULTS}

Different measurements have been carried out to experimentally characterize and verify the feasibility of the proposed transmitting architecture. For the characterization, the OBFN demonstrator was connected, using fiber patchcords, to a corporate fed $8 \times 8$ elements array with 8 independent inputs in each 8 -element row subarray (Fig. 10). The antenna (Fig. 11) is in fact an $8 \times 8$ phased array where each set of 8 vertical

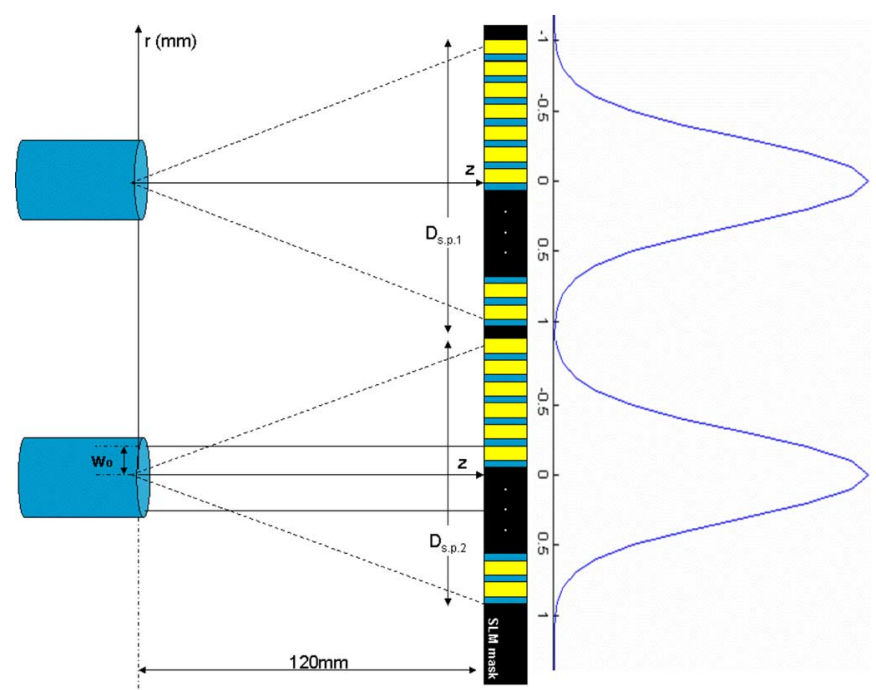

Fig. 9. Light beam incidence on the "super-pixeled" configured PAL-SLM.

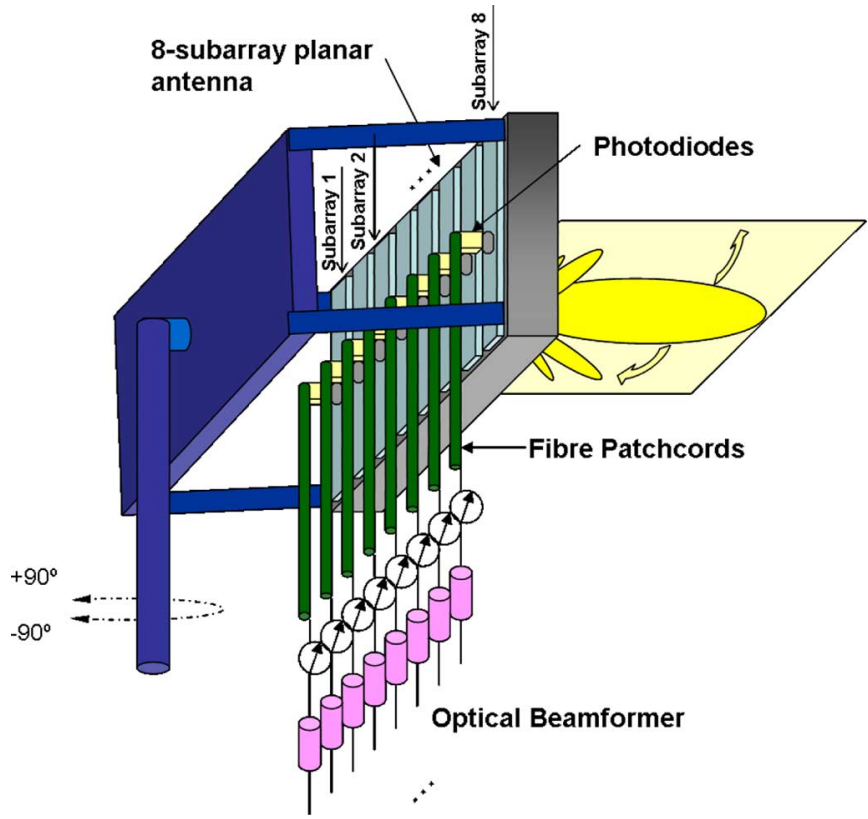

Fig. 10. Schematic representation of the array connection to the 8 photodiodes.

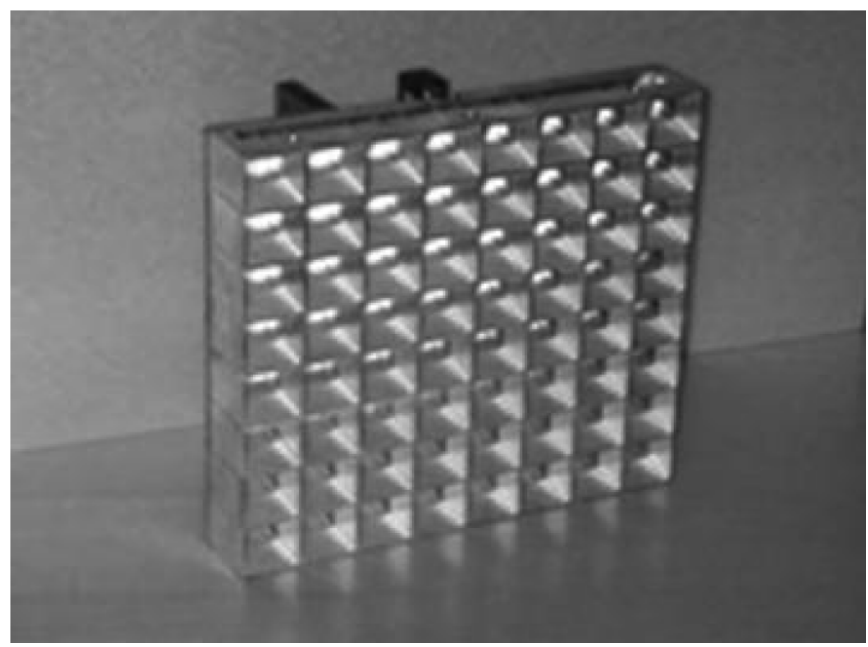

Fig. 11. Picture of the $8 \times 8$ element antenna array. 


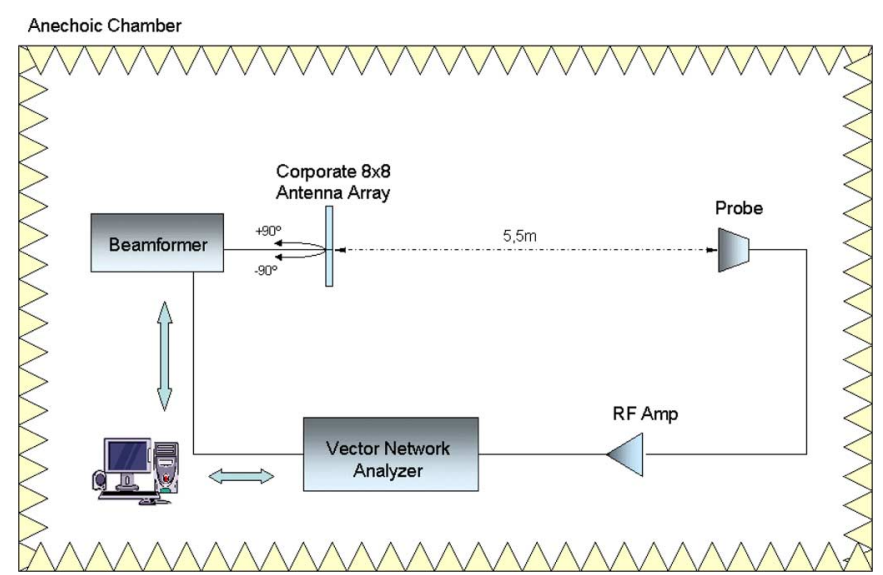

Fig. 12. Scheme of the experimental setup for the antenna radiation pattern measurement.

elements, having an inter-element spacing of $0.8 \lambda$, was internally connected as a corporate array element, configuring a subarray, to produce fan-beams. The whole system was placed properly in an anechoic chamber with approximate dimensions of $(7 \times 7 \times 10) \mathrm{m}^{3}$. The receiving antenna, consisting into a calibrated pyramidal $\mathrm{X}$-band horn, was fixed-beyond the far-field distance-at $5.5 \mathrm{~m}$ away from the transmitting antenna, while OBFN antenna was rotated to measure radiation patterns, as can be seen from Fig. 12.

Additionally, for the frequency of $8 \mathrm{GHz}$, a reconstruction of the amplitude and phase of the current along each subarray antenna element was performed for different beam steering cases. This reconstruction is based on the Fourier transform relation between antenna current distribution and far-field radiation pattern [25]. The plane wave spectrum (PWS) of the radiated fields, being the Fourier Transform of the antenna current distribution, may be extracted from the far field spherical measurement of the antenna radiation pattern. Thus, performing an inverse Fourier Transform of the measured radiation pattern, the equivalent current on the antenna elements can be reconstructed.

The beamforming architecture, depicted in Fig. 2, is initially fed with two lasers with wavelengths 1550.92 and $1552.52 \mathrm{~nm}$ used as optical sources. The lasers are set to the output power level of $5 \mathrm{dBm}$ and $7 \mathrm{dBm}$, respectively, to obtain similar powers at the end of the system. Both signals are amplitude modulated using two dual drive-MZMs (DD-MZMs), fed by the signal generated by an RF vector network analyzer. Both SSB signals are combined using an optical coupler and launched to the DGD module and then to a compact EDFA. After optical amplification, both modulated optical carriers are demultiplexed using an optical $1 \times 2$ demultiplexer and again each channel is split in four channels (to feed the 8 subarray antenna). Then, polarization controllers are used to adjust the polarization state of the optical signals to the axis of the SLM. To control the phase shift of the RF signals, a PAL-SLM of $1 \times 128$ pixels and a free-space polarizer are employed prior to a set of 7 pairs of collimators (7 inputs -7 outputs) used to collimate the seven of the eight beams, up to $11 \mathrm{~cm}$ distance. To control the amplitude of the optical signals a set of VOAs is included in the setup. Eight fiber patchcords of 5 meters are used to remotely feed the 8 element

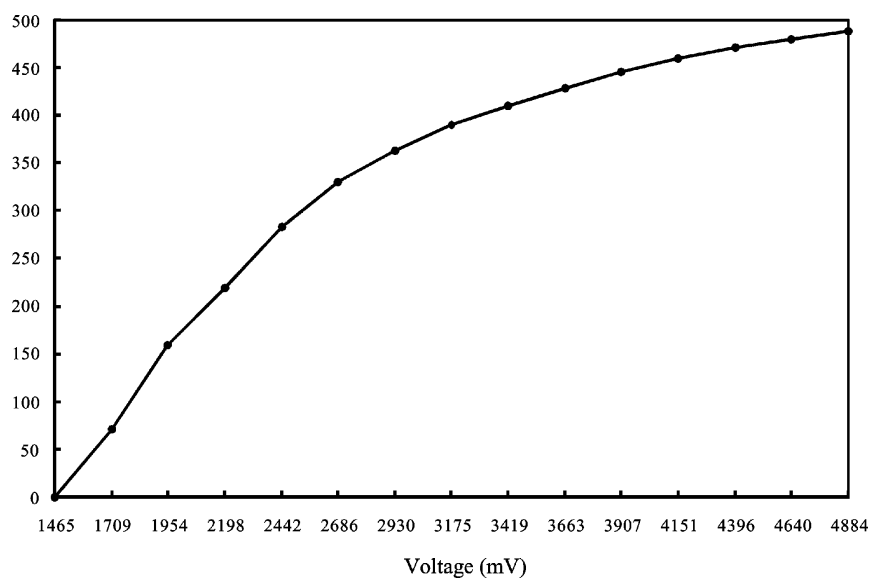

Fig. 13. Phase modulation obtained over $8 \mathrm{GHz}$ signal for one beamformer channel.

subarray planar antenna while a set of eight photodiodes is directly connected to the antenna elements (see Fig. 10). Finally, the set-up of Fig. 12 is used to measure the radiation patterns.

\section{A. Calibration}

In order to carry out the measurements and provide accurate and precise results, several adjustments have to be done. First, it has to be assured the $90^{\circ}$ phase lag between MZM drives in order to ensure SSB modulation and thus fully exploit the phase control capability of the PAL-SLM [19]. Second, the adequate state of polarization has to be established at the entrance of the DGD and the PAL-SLM, by means of the polarization controllers. Finally, the calibration curves (i.e., the phase shift versus voltage drive level, as shown in Fig. 13) of each channel have to be determined. As seen from Fig. 13, using the PAL-SLM the phase of an $8 \mathrm{GHz}$ tone can be controlled over a range of $3 \pi$.

\section{B. Beam Steering Capability}

Radiation patterns for the 8-subarray antenna have been measured for different beam steering angles at five frequencies between 7.5 and $8.5 \mathrm{GHz}$. As an example, Fig. 14 shows the results from the measurement of the antenna radiation pattern with the main lobe pointing at $-20^{\circ}$ with respect to the broadside. The beam squint observed agrees with the theoretically predicted value $\left(1.2^{\circ}\right.$ at $7.5 \mathrm{GHz}$ and $1.4^{\circ}$ at $\left.8.5 \mathrm{GHz}\right)$ and the SLR is $13 \mathrm{~dB}$ as approximately foreseen theoretically. Fig. 15 represents the reconstruction of the current amplitudes (Fig. 15, leftmost) and phases (Fig. 15, rightmost). Amplitude deviations are observed to be in the order of $\pm 2 \mathrm{~dB}$, mainly due to the amplitude frequency dispersion produced by the PAL-SLM and VOA, while a good linear phase behavior is obtained.

\section{Multibeam Capability}

To demonstrate the multibeam capability of the system the PAL-SLM is configured to provide two sets of phase shifts for two 4-element beam arrays; one for the antenna subarrays 1 to 4 and the other one for the antenna subarrays 5 to 8 . Thus, the drive levels have been adjusted for each channel to obtain the first 4-element array pointing at a fixed scan angle and the second 


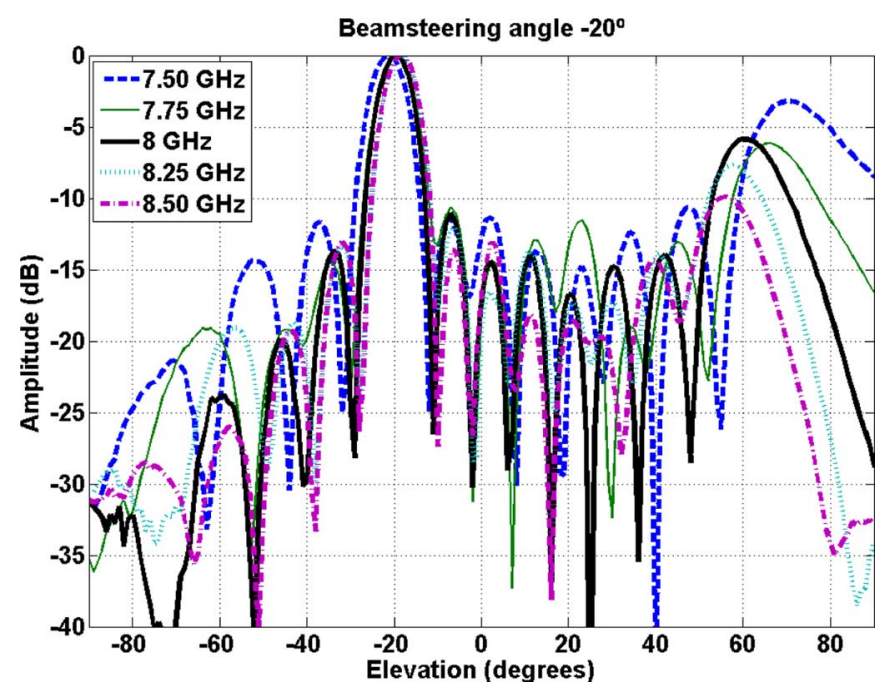

Fig. 14. Radiation pattern of the optically controlled phased array antenna steered at $-20^{\circ}$.

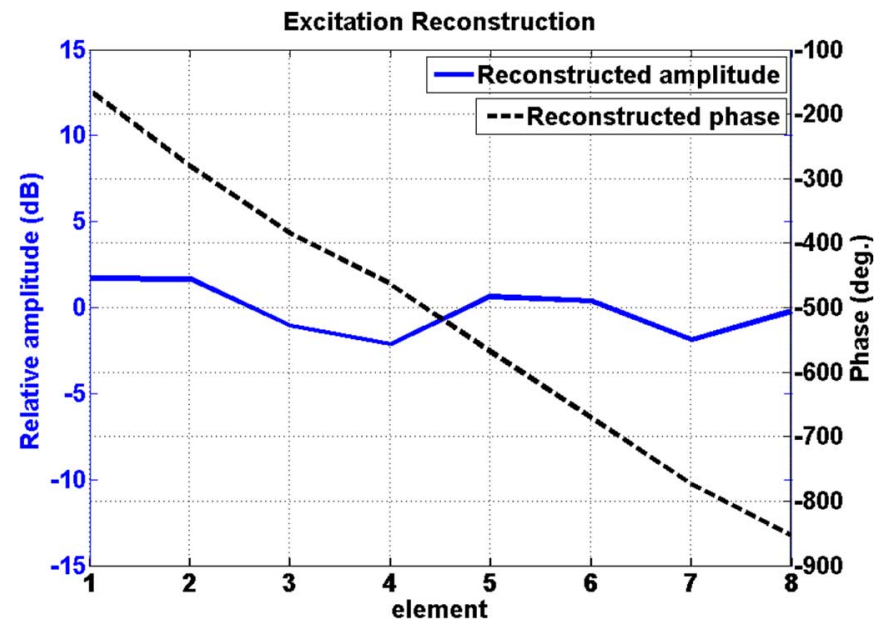

Fig. 15. Reconstruction of the currents amplitude and phase for the case of $-20^{\circ}$ at $8 \mathrm{GHz}$.

4-element array pointing at a different angle, at the frequency of $8 \mathrm{GHz}$. To clearly observe the two different far-field patterns they have been measured radiating firstly only with the first set of four elements (beam A, Fig. 16) and secondly, only with the second set of four elements (beam B, Fig. 17). The measurements have been carried out at the same RF frequency $(8 \mathrm{GHz})$ although beams at different frequencies are also possible. Beam A was adjusted to point to $-15^{\circ}$ and beam $\mathrm{B}$ to $20^{\circ}$. As can be seen from Figs. 16 and 17 the extracted radiation patterns agree quite well with theory.

\section{Tapering Distribution Capability}

Furthermore, the tapering distribution capability by means of the VOAs, in order to reduce the sidelobe level, has been demonstrated. Fig. 18 shows the radiation pattern for a triangular distribution of currents (Table II) on the antenna elements, in order to increase the SLR. Measurements at the operating frequency of 8 $\mathrm{GHz}$, demonstrate an increase of the SLR of up to approximately $20 \mathrm{~dB}$ with respect to the previous uniform array distribution.

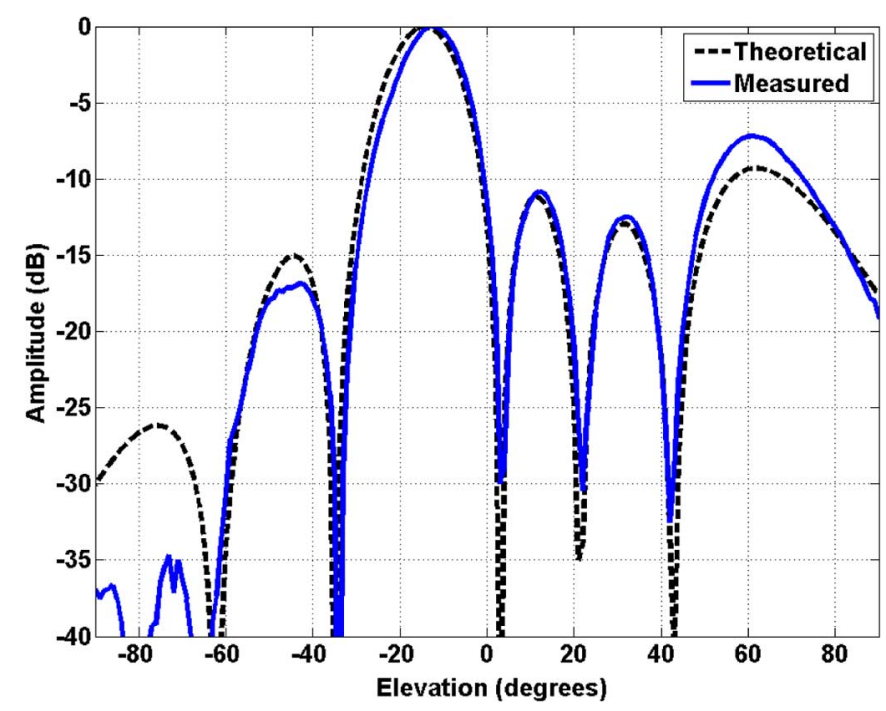

Fig. 16. Radiation pattern for the multibeam operation $\left(-15^{\circ}, 20^{\circ}\right)$ and beam A: (dashed) theoretical, (solid) measured.

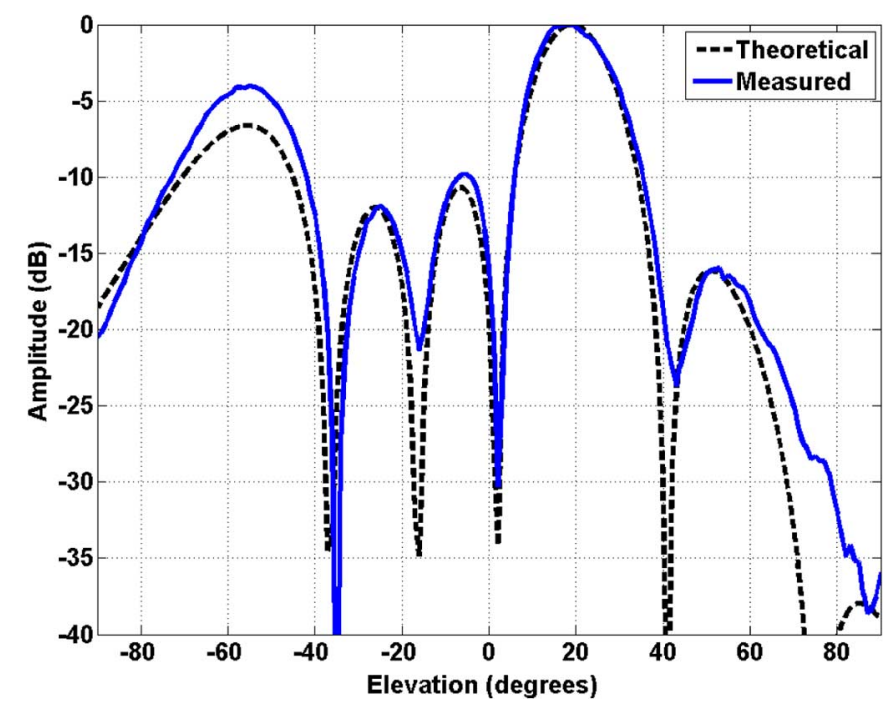

Fig. 17. Radiation pattern for the multibeam operation $\left(-15^{\circ}, 20^{\circ}\right)$ and beam B: (dashed) theoretical, (solid) measured.

\section{E. TTD Capability}

Finally, the wideband system has been checked for its directional pointing stability when using time delays instead of phase shifts, where [26] beam squint problem can be highly reduced.

Measurements have taken place for the case of the beam steering at $15^{\circ}$, showing $1 \mathrm{GHz}$ wide bandwidth operation. Fig. 19 represents the resulted radiation patterns, for the frequencies of $7.5,8$, and $8.5 \mathrm{GHz}$. The measurements have been repeated for the same beam steering case, with the phase control performed by the PAL-SLM. Both cases are illustrated in Fig. 20. As can be seen from the figure, the beam steering angle variability with frequency is clearly more stable when employing only TTD (from $18^{\circ}$ to $18.5^{\circ}$, when frequency sweeps from 7.5 to $8.5 \mathrm{GHz}$, Fig. 20/ Right) than for the phase shifter case (from $14.4^{\circ}$ to $16.9^{\circ}$, when frequency sweeps from 7.5 to $8.5 \mathrm{GHz}$, Fig. 20/ Left). 


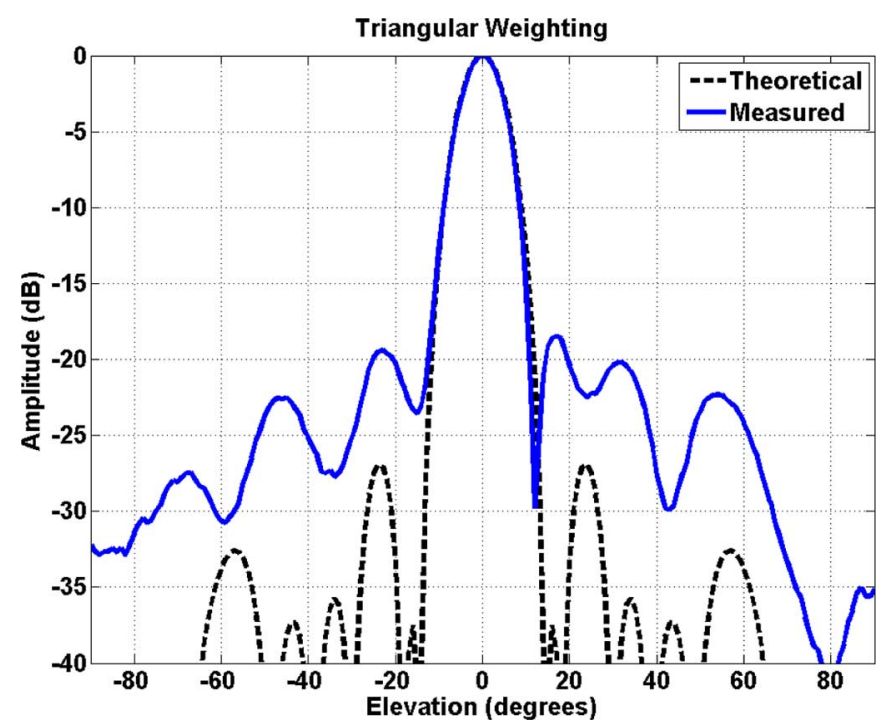

Fig. 18. Radiation pattern of the beamformer at $8 \mathrm{GHz}$, for the broadside case when a triangular tapering is used.

TABLE II

WEIGHTING COEFFICIENTS FOR TRIANGULAR DISTRIBUTION

\begin{tabular}{|l|c|c|c|c|c|c|c|c|}
\hline Channels & $\mathbf{1}$ & $\mathbf{2}$ & $\mathbf{3}$ & $\mathbf{4}$ & $\mathbf{5}$ & $\mathbf{6}$ & $\mathbf{7}$ & $\mathbf{8}$ \\
\hline Linear & 0.25 & 0.5 & 0.75 & $\mathbf{1}$ & $\mathbf{1}$ & 0.75 & 0.5 & 0.25 \\
\hline $\begin{array}{l}\text { Relative } \\
\text { Amplitude } \\
\text { (dB) }\end{array}$ & $\mathbf{- 1 2}$ & $\mathbf{- 6 . 0 2}$ & $\mathbf{- 2 . 5}$ & 0 & 0 & $\mathbf{- 2 . 5}$ & $\mathbf{- 6 . 0 2}$ & $\mathbf{- 1 2}$ \\
\hline
\end{tabular}

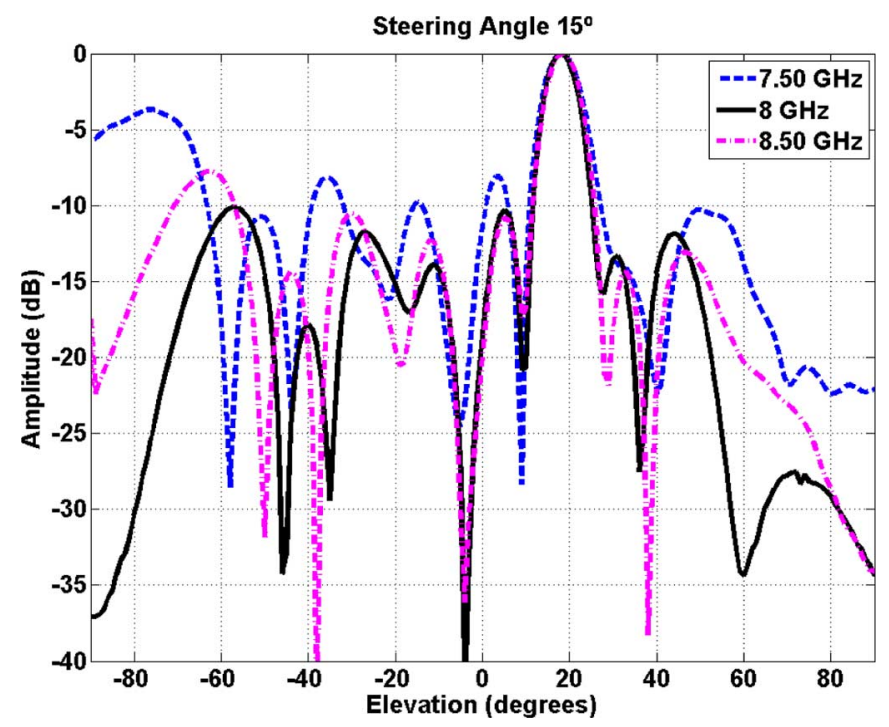

Fig. 19. Radiation pattern for the case of steering at $15^{\circ}$ by the adequate adjustment of the time delays (having removed the PAL-SLM and the DGD).

\section{CONCLUSION}

The performance of an optical beamforming network based on fiber-optic delay lines and a spatial light modulator phase shifter has been experimentally demonstrated, showing great potential for present and future wideband applications. In a
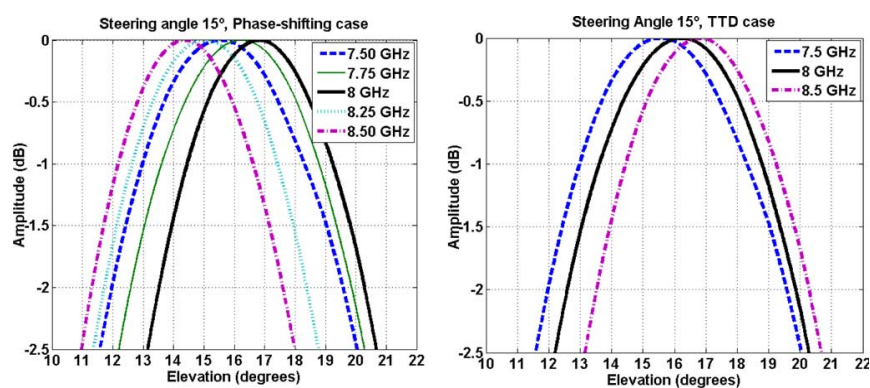

Fig. 20. Left: Main lobe for the case of steering at $15^{\circ}$ with phase-shifting. Right: Main lobe for the case of steering at $15^{\circ}$ with TTD.

practical implementation, different factors (e.g., losses, SNR, crosstalk, stability etc.) need to be taken into account. The capability of beam steering, tapering distribution for the reduction of the sidelobe level, multibeam operation and TTD performance has been demonstrated and far-field radiation patterns for an 8 -subarray antenna at the $\mathrm{X}$-band have been measured, proving its feasibility and effectiveness. Consequently, the combination of free-space components (SLMs) and optical fiber devices (as ODLs) is considered a good solution for the implementation of wideband phased antenna arrays.

\section{REFERENCES}

[1] P. Angeletti, M. Lisi, and R. Mizzoni, "Antennas for broadband and mobile satellite communications," in Proc. IEEE Antenna and Propagation Society Int. Symp., 2002, vol. 4, pp. 736-739.

[2] Cassini-Huygens. Mission to Saturn and Titan [Online]. Available: http://saturn.jpl.nasa.gov/spacecraft/index.cfm

[3] Telkom-2 [Online]. Available: http://www.orbital.com/SatellitesSpace/GEO/TELKOM/index.html

[4] M. Lisi, G. Panariello, and V. Santachiara, "An active phased-array antenna at X-band for spaceborne SAR applications: System design tradeoffs," in Proc. 8th Int. Conf. on Antennas and Propagation, 1993, vol. 1, pp. 614-617.

[5] J. H. G. Ender and A. R. Brenner, "PAMIR-A wideband phase array SAR/MTI system," in Proc. EUSAR Conf., Cologne, Germany, Jun. 4-6, 2002, pp. 157-162.

[6] H.-M. J. Cantalloube and P. Dubois-Fernandez, "Airborne X-band SAR imaging with $10 \mathrm{~cm}$ resolution-Technical challenge and preliminary results," in Proc. IEEE/IGARSS, Toulouse, France, Jul. 21-25, 2003, pp. 533-535.

[7] European Space Agency, in Proc. ENVISAT Symp., Noordwijk, The Netherlands, Apr. 23-27, 2007.

[8] R. D. Esman, M. Y. Frankel, J. L. Dexter, L. Goldberg, and M. G. Parent, "Fiber optic prism true time delay antenna feed," IEEE Photon. Technol. Lett., vol. 5, pp. 1347-1349, Nov. 1993.

[9] B. Vidal, J. L. Corral, M. A. Piqueras, and J. Martí, "Optical delay line based on arrayed waveguide gratings' spectral periodicity and dispersive media for antenna beamforming applications," IEEE $J$. Sel. Topics Quantum Electron., vol. 8, pp. 1202-1210, Nov./Dec. 2002.

[10] B. Vidal, J. L. Corral, and J. Martí, "Optical delay line employing an arrayed waveguide grating in fold-back configuration," IEEE Microw. Wireless Compon. Lett., vol. 13, pp. 238-240, Jul. 2003.

[11] D. T. K. Tong and M. C. Wu, "A novel multiwavelength optically controlled phased array antenna with a programmable dispersion matrix," IEEE Photon. Technol. Lett., vol. 8, pp. 812-814, Jun. 1996.

[12] M. A. Piqueras, G. Grosskopf, B. Vidal, J. Herrera, P. Sanchis, V. Polo, J. L. Corral, A. Marceaux, J. Galière, J. Lopez, A. Enard, J. L. Valard, O. Parillaud, E. Estèbe, N. Vodjdani, J. H. d. Besten, F. Soares, M. K. Smit, and J. Marti, "Optically beamformed beamswitched adaptive antennas for fixed and mobile broadband wireless access networks," IEEE Trans. Microw. Theory Tech., vol. 54, pp. 887-899, Feb. 2006. 
[13] N. A. Riza, S. A. Khan, and M. A. Arain, "Flexible beamforming for optically controlled phased array antenna," Opt. Commun., vol. 227, pp. 301-310, Sep. 2003.

[14] D. Dolfi, F. Michel-Gabriel, S. Bann, and J. P. Huignard, "Two-dimensional optical architecture for time-delay beam forming in a phasedarray antenna," Opt. Lett., vol. 16, pp. 225-257, Feb. 1991.

[15] Project OBEFONE: Technologies for Optical Payloads ESA-SENER TRP EE-010.

[16] F. Dalmases, S. Blanch, J. Romeu, L. Jofre, B. Vidal, J. Marti, I. Mackenzie, E. Vez, and J. Santamaria, "Optically beamformed array performance," in Eur. Conf. on Antennas and Propagation, EuCAP, Nice, France, Nov. 6-11, 2006.

[17] F. Dalmases, S. Blanch, J. Romeu, L. Jofre, B. Vidal, J. Marti, I. Mackenzie, E. Vez, and J. Santamaria, "Wideband optical TTD SAR antenna," in IEEE Mediterranean Electrotechnical Conf-_Melecon, Malaga, Spain, May 16-19, , pp. 336-339.

[18] B. Vidal, T. Mengual, C. Ibáñez-López, and J. Martí, "Optical beamforming network based on fiber optical delay lines and spatial light modulators for large antenna arrays," IEEE Photon. Technol. Lett., vol. 18, no. 24, pp. 2590-2592, Dec. 2006.

[19] T. Mengual, B. Vidal, C. Stoltidou, S. Blanch, J. Marti, L. Jofre, I. McKenzie, and J. M. del Cura, "Optical phase-based beamformer using MZM SSB modulation combined with crystal polarization optics and a spatial light modulator," Optics Communications, accepted for publication.

[20] U. Efron, Spatial Light Modulator Technology, M. Dekker, Ed. Boca Raton, FL: CRC Press, 1995.

[21] A.-A. Abdul, Photonics. Principles and Practices. Boca Raton, FL: CRC Press, 2006.

[22] C. D. Richard, The Electrical Engineering Handbook, 2nd ed. Boca Raton, FL: CRC Press, 1997.

[23] J. Romeu, L. Jofre, and A. Cardama, "An approximate expression to estimate signal-to-noise ratio improvement in cylindrical near-field measurements," IEEE trans. on Antennas and Propagation, vol. 42, no. 7, pp. 1007-1010, Jul. 1994.

[24] C. G. Lal, Smart Antennas. Boca Raton, FL: CRC Press, 2004, pp. 23-25.

[25] S. Blanch, L. Jofre, and J. Romeu, "Comparison between classical and equivalent current approach near-field to far-field transformation," in Proc. Int. Symp. Antennas and Propagation Society, 1995, vol. 1, pp. 260-263.

[26] W. Wulf-Dieter, Radar Techniques Using Array Antennas. Piscataway, NJ: IEEE Publications, 2001.

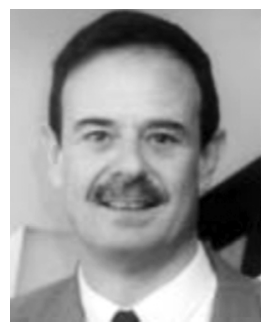

Lluís Jofre (S'79-M'83-SM'07) was born in Mataró, Spain, in 1956. He received the M.Sc. (Ing.) and Ph.D. (Doctor Ing.) degrees in electrical engineering, from the Technical University of Catalonia (UPC), Barcelona, Spain, in 1978 and 1982, respectively.

From 1979 to 1980, he was Research Assistant in the Electrophysics Group at UPC, where he worked on the analysis and near field measurement of antenna and scatterers. From 1981 to 1982, he joined the Ecole Superieure d'Electricite, Paris, France, where he was involved in microwave antenna design and imaging techniques for medical and industrial applications. In 1982, he was appointed Associate Professor at the Communications Department of the Telecommunication Engineering School, UPC, where he became Full Professor in 1989. From 1986 to 1987, he was a Visiting Fulbright Scholar at the Georgia Institute of Technology, Atlanta, working on antennas, and electromagnetic imaging and visualization. From 1989 to 1994, he served as Director of the Telecommunication Engineering School, UPC, and from 1994 to 2000, as UPC Vice-rector for Academic Planning. From 2000 to 2001, he was a Visiting Professor at the Electrical and Computer Engineering Department, Henry Samueli School of Engineering, University of California. From 2002 to 2004, he served as Director of the Catalan Research Foundation and, since 2003, as Director of the UPC-Telefonica Chair. His research interests include antennas, electromagnetic scattering and imaging, and system miniaturization for wireless and sensing industrial and bio applications. He has published more than 100 scientific and technical papers, reports and chapters in specialized volumes.

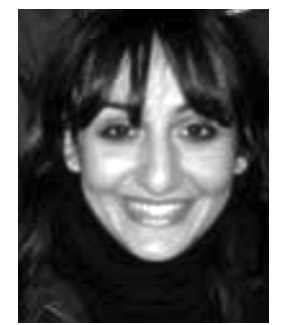

Chrysavgi Stoltidou was born in Thessaloniki, Greece, in 1980. She received the Master Engineering diploma in electrical and computer engineering (telecommunications specialty) from the Technical School of the Aristotle University of Thessaloniki, where, from September 2004 to March 2005, she completed her diploma thesis on the topic of spectral losses in optical fibers. She received the M.Sci. degree in information and communication technologies from the Technical University of Catalonia (UPC), Barcelona, Spain, in July 2007.

From 2005 until January 2008, she worked as a Research Assistant in the Department of Signal Theory and Communications, UPC, working under the topic of wideband phased array antennas. She is now with Consulting Ltd., Madrid, Spain. Her research interests include antennas, optical beamforming networks and plasma surface structures.

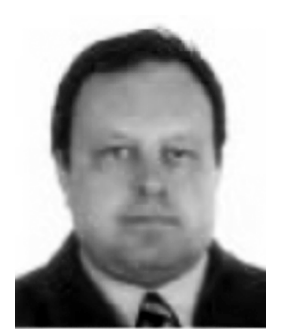

Sebastián Blanch was born in Barcelona, Spain, in 1961. He received the Ingeniero and Doctor Ingeniero degrees in telecommunication engineering, both from the Polytechnic University of Catalonia (UPC), Barcelona, Spain, in 1989 and 1996, respectively.

In 1989, he joined the Electromagnetic and Photonics Engineering Group, Department of Signal Theory and Communications, UPC, where he is currently an Associate Professor. His research interests include near-field antenna measurements and imaging, antenna diagnostics, and antenna design.

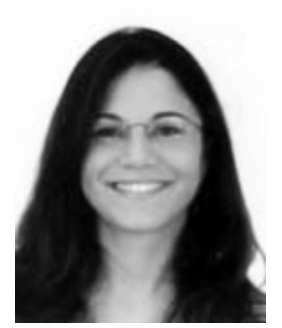

Teresa Mengual was born in Valencia, Spain, in 1979. She received the M.Sc. degree in physics, from the University of Valencia, Valencia, Spain, in 2003.

In 2005, she joined the Nanophotonics Technology Center, Universidad Politécnica de Valencia, Spain, where she is working toward the $\mathrm{Ph} . \mathrm{D}$. degree. Her research interests include free space optics, optical beamforming and signal processing

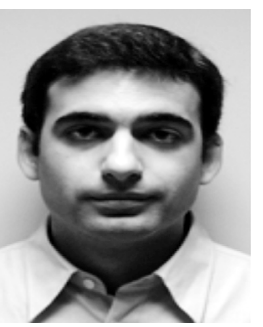

Borja Vidal was born in Alicante, Spain, on April 24, 1978. He received the Ingeniero de Telecomunicación degree and the Doctor Ingeniero (Ph.D.) degree both from the Universidad Politécnica de Valencia, Valencia, Spain, in 2001 and 2004, respectively.

In 2005, he joined the Universidad Politécnica de Valencia where he currently he an Assistant Professor. His research interests include phased array antennas, optical beamforming networks, photonic microwave filters and microwave and millimetrewave optical fibre systems. He has authored or coauthored over 25 journal publications and 30 conference presentations.

Dr. Vidal received the IEEE Lasers and Electro-Optics Society (LEOS) Graduate Student Fellowship in 2004. 


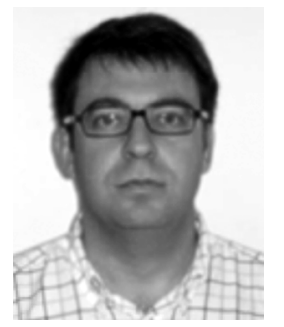

Javier Martí (S'89-M'92) received the Ingeniero de Telecomunicación degree from the Universidad Politécnica de Catalunya, Barcelona, Spain, and the Doctor Ingeniero de Telecomunicación degree (Ph.D.) from the Universidad Politécnica de Valencia, Valencia, Spain, in 1991 and 1994, respectively.

In 1989 and 1990, he was an Assistant Lecturer with the Escuela Universitaria de Vilanova, Barcelona. From 1991 to 2000, he was a Lecturer and an Associate Professor with the Telecommunication Engineering Faculty, Universidad Politécnica de Valencia, where he is currently a Full Professor and, since 2003, he has been the Director of the Valencia Nanophotonics Technology Center. He has authored or coauthored over 200 papers in referred international technical journals and holds 12 patents in the fields of broadband hybrid fiber-radio systems and microwave/millimeterwave photonics, fiber-based access networks, terabit/s OTDM/WDM optical networks, advanced optical processing techniques for microwave signals, ultrahigh speed data transmission, and (silicon) integrated photonics, including planar photonic crystals and metamaterials. He has participated very actively in the European Research Framework (FP5 and FP6), leading several projects in the areas of broadband radio-over-fiber access (OBANET, GANDALF), alloptical processing in photonic networks (LASAGNE), and nanophotonic logic gates (PHOLOGIC).
Prof. Martí has served as member of the Technical Program Committee of ECOC, the IEEE Lasers and Electro-Optics Society (LEOS), Microwave Photonics, and other international workshops and conferences.

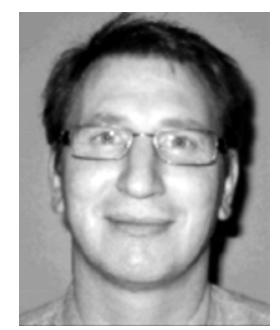

Iain McKenzie received the B.Eng. degree in electronic and electrical engineering and the M.Phil. degree in optoelectronics from the University of Strathclyde, Glasgow, U.K., in 1991 and 1993, respectively.

Since 2002, he has been working as a Contractor with the European Space Agency, Noordwijk, The Netherlands, in the Optoelectronic Section of The Directorate of Technical and Quality Management, ESTEC. His research interests include optical communications, optical-fiber sensors, microphotonics, and optoelectronic packaging for harsh environments.

J. M. del Cura, photograph and biography not available at the time of publication. 